\title{
Clinacanthus nutans: Its potential against diabetic vascular diseases
}

\author{
Ahmad Khusairi Azemi ${ }^{1}$, Siti Safiah Mokhtar ${ }^{\circledR 1}$, Aida Hanum Ghulam Rasool ${ }^{1,2^{*}}$ \\ ${ }^{1}$ Department of Pharmacology, School of Medical Sciences, Universiti Sains Malaysia, \\ Health Campus, 16150 Kota Bharu, Kelantan, Malaysia, ${ }^{2}$ Hospital Universiti Sains Malaysia, \\ Universiti Sains Malaysia, Health Campus, 16150 Kota Bharu, Kelantan, Malaysia
}

\begin{abstract}
Diabetes is an independent risk factor for the development of cardiovascular disease, with approximately $80 \%$ of cardiovascular mortality and morbidity linked to vascular complications such as atherosclerosis. It has been estimated that up to one-third of patients with diabetes mellitus use some form of complementary and/or alternative medicine. One plant that has received attention from diabetic patients for its perceived antidiabetic properties is Clinacanthus nutans, a member of the Acanthaceae family that is known as snake grass. Ethnomedical applications of this herb have been identified for the treatment of certain conditions, including fever, diabetes, skin rashes, and insect bites. This review aims to assess the potential of $C$. nutans to be used in the prevention and/ or treatment of diabetic vasculopathy. Evidence for antidiabetic, anti-inflammatory, and dyslipidemic properties of $C$. nutans, as shown from experimental studies, is presented and discussed. Diabetes, inflammation, and hyperlipidemia are known to play significant roles in the initiation and severity of diabetic cardiovascular disease; thus, targeting these factors might be beneficial for preventing and/ or treating diabetic vasculopathy.
\end{abstract}

Keywords: C. nutans. Diabetes. Inflammation. Cardiovascular disease

\section{INTRODUCTION}

Diabetes mellitus is a metabolic disorder characterized by altered glucose and lipid metabolism, which leads to persistent hyperglycemia. Individuals with diabetes have a greater risk of developing vascular complications despite intensive glycemic control, stressing the need for novel approaches to lessen the burden of diabetic-mediated macrovascular injury. The vascular endothelium plays a significant role in diabetes-associated atherosclerosis through the regulation of vessel permeability, inflammation, coordination of leukocyte trafficking, and thrombosis (Funk et al., 2012; Kolka et al., 2013; Sharma et al.,

*Correspondence: A. H. G. Rasool. Department of Pharmacology. School of Medical Sciences. Universiti Sains Malaysia (Health Campus). 16150 Kota Bharu, Kelantan, Malaysia. Phone: 609-7676123. Fax: 609-7653370. E-mail: aida.rasool@yahoo.com
2012). Endothelial cells regulate vascular function and structure because of their strategic anatomic position between the vessel wall and circulating blood. In normal endothelial cells, biologically active substances are synthesized and released to maintain nutrient delivery and vascular homeostasis and to ensure adequate blood flow, while also preventing leukocyte extravasation and thrombosis.

Nitric oxide (NO) is produced by endothelial NO synthase (eNOS) in the endothelial cells through fiveelectron oxidation of the guanidine-nitrogen terminal L-arginine. NO causes vasodilation by activating guanylyl cyclase on subjacent vascular smooth muscle cells (Creager et al., 2003). NO also protects blood vessels from endogenous injury by mediating molecular signals that inhibit leukocyte and platelet interaction with the vascular wall and prevent vascular smooth muscle proliferation and migration (Kubes et al., 1991; 
Radomski et al., 1987; Sarkar et al., 1996). A reduction in endothelium-derived NO increases the activity of the pro-inflammatory transcription factor nuclear factor-kappa B (NF-кB), resulting in over-expression of leukocyte adhesion molecules and an increase in the production of cytokines and chemokines. These activities promote vascular smooth muscle cell and monocyte migration into intima and the formation of foam cells, characterizing the initial morphological changes of atherosclerosis (Creager et al., 2003). Endothelial dysfunction, as characterized by impaired endothelium-dependent NO-mediated relaxation, has been reported in both cellular and experimental models of diabetes (Mokhtar et al., 2016). In addition, endothelial dysfunction, diabetes, and atherosclerosis are linked to a heightened state of oxidative stress and its main mediator, superoxide anion. Superoxide scavenges free NO to form peroxynitrite, another potent reactive oxygen species (ROS), which significantly contributes to lower NO bioavailability (Sharma et al., 2012). A majority of the metabolic derangement known to occur in diabetes, including hyperglycemia, insulin resistance, and excess free fatty acid liberation, mediate abnormalities in endothelial cell function by affecting the synthesis or degradation of NO (Creager et al., 2003; King, 1996) (Figure 1).

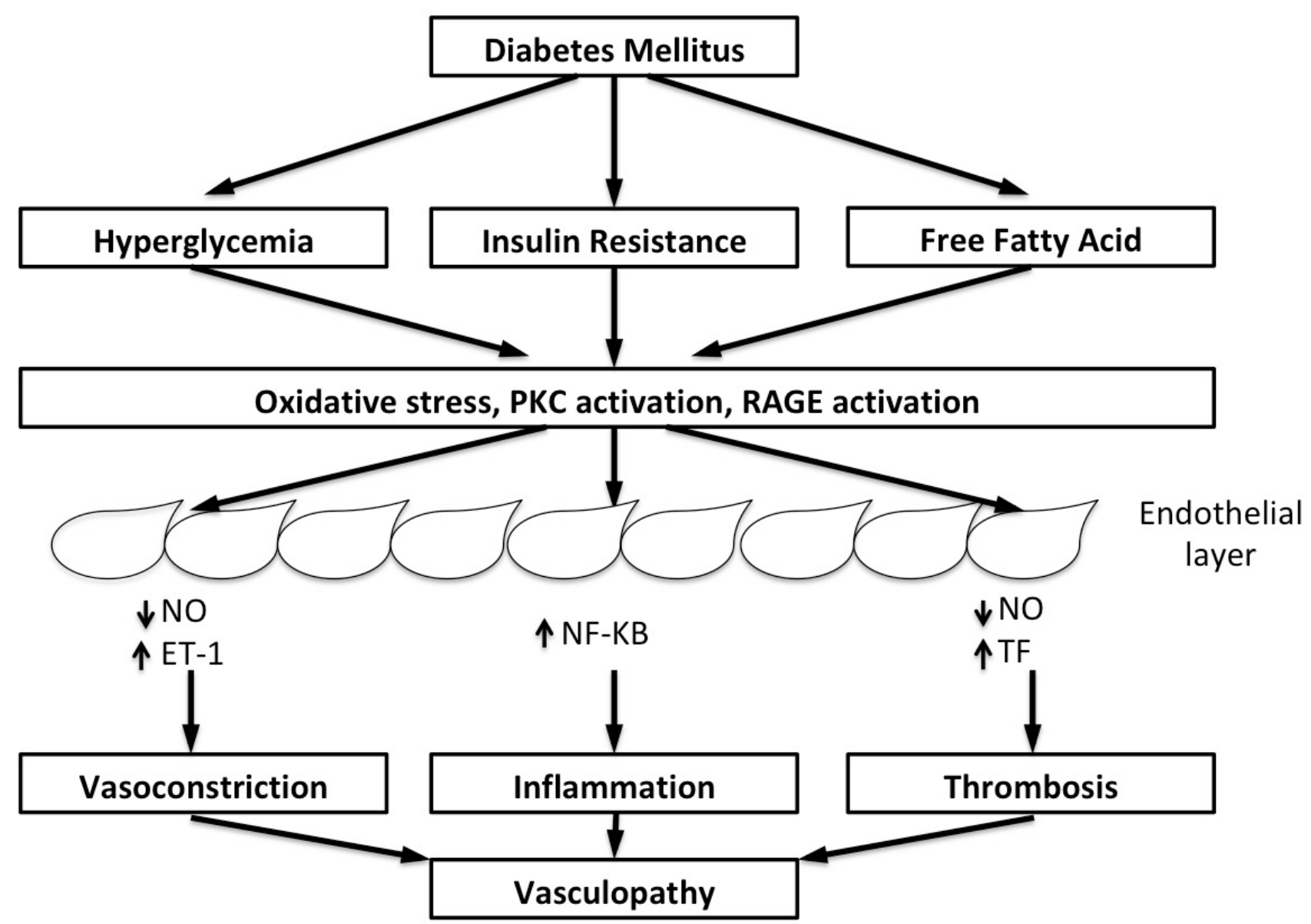

FIGURE 1 - The metabolic abnormalities that characterize diabetes, particularly hyperglycemia, insulin resistance, and free fatty acids, act to stimulate molecular mechanisms that change the function and structure of blood vessels. These include increased oxidative stress, disturbances of intracellular signal transduction (activation of protein kinase $\mathrm{C}$ [PKC]), and activation of receptors for advanced glycation end product (RAGE). Consequently, there is decreased availability of NO, increased production of endothelin (ET-1), activation of transcription factors such as NF- $\mathrm{B}$, and production of prothrombotic factors such as tissue factor (TF). 
Insulin resistance characterizes type 2 diabetes mellitus. Insulin increases NO production in endothelial cells by increasing the activity of NO synthase (NOS) via activation of Akt kinase and phosphatidylinositol-3. Thus, insulin increases endothelium-dependent vasodilation. In diabetic subjects, insulin resistance impairs the phosphatidylinositol-3 kinase pathway and causes a reduction in insulin's ability to activate NOS and produce NO (Creager et al., 2003). In addition, insulin resistance is associated with elevations of free fatty acid levels. In the diabetic state, levels of free fatty acids are elevated because of their excess liberation from adipose tissue and diminished uptake by skeletal muscle (Boden, 1999; Creager et al., 2003; Kelley, Simoneau, 1994). High free fatty acid levels cause endothelial dysfunction through several mechanisms, including activation of $\mathrm{PKC}$, increased production of oxygen-derived free radicals, and exacerbation of dyslipidemia (Creager et al., 2003; Dresner et al., 1999; Inoguchi et al., 2000). It has been proven that free fatty acids reduce endotheliumdependent vasodilation in human and animal models in vivo (Creager et al., 2003; Steinberg et al., 1997), and elevation of free fatty acid concentrations activate PKC and decrease insulin receptor substrate-1 associated phosphatidylinositol-3 kinase activity (Creager et al., 2003; Dresner et al., 1999; Griffin et al., 1999). These effects reduce NOS activity, as discussed above.

\section{Ethnopharmacology of C. nutans}

Clinacanthus nutans (Burm. f.) Lindau, commonly called Sabah Snake Grass, or "Belalai Gajah" in layman's terms, is widely used in Malaysia, Thailand, and Indonesia as a traditional medicine. The name Sabah Snake Grass is derived from its application by traditional practitioners as an antivenin for poisonous snakes (Kamarudin et al., 2017). Traditionally, it has been used to treat diabetes, cancer, herpes infections, inflammation, and various skin problems (Alam et al., 2017; Sakdarat et al., 2006; Sangkitporn et al., 1995; Shim et al., 2013). In Malaysia, the fresh leaves of $C$. nutans are commonly consumed as an herbal tea for the treatment of diabetes, skin rashes, and fever, as well as to induce diuresis (Kamarudin et al., 2017; Shim et al., 2013). In addition, the fresh leaves of $C$. nutans are consumed in the treatment of insect bites (ant, bee, hornet, mosquito, and wasp), stings by Chilopoda (centipede) and Arachnida (scorpion), sea creature bites (catfish and jellyfish), snake bites, eye diseases, and allergic responses (Daduang et al., 2008; Kamarudin et al., 2017; Uawonggul et al., 1986).

\section{Bioactivities of C. nutans}

Natural products continue to be one of the major sources of drug discoveries owing to their ability to evoke complex cellular pathways in preventing and alleviating various diseases (Kamarudin et al., 2017). Therefore, research of natural products for chronic diseases is of major significance as it may lead to the development of newer therapeutic agents with potentially fewer side effects. Previous studies and scientific investigations have supported the traditional use of $C$. nutans in various diseases. Scientifically, extracts of $C$. nutans are reported to have a number of pharmacological properties, with effects that include being antihyperglycemic (Alam et al., 2017), antibacterial (Huang et al., 2015), antiinflammatory (Ho et al., 2013; Wanikiat et al., 2008), anti-herpes (Kunsorn et al., 2013; Wanikiat et al., 2008), antioxidant (Sangkitporn et al., 1995; Sarega et al., 2016), and antihyperlipidemic (Sarega et al., 2016).

\section{Pharmacologic Properties of C. nutans That Are Potentially Useful for Preventing or Ameliorating Diabetic Vasculopathy}

\section{Antihyperglycemic effect}

Diabetes is an endocrine disease characterized by hyperglycemia arising from insulin insufficiency and/ or insulin resistance. In type 2 diabetes, macrovascular and microvascular complications are associated more with postprandial hyperglycemia than with fasting glucose levels. Postprandial hyperglycemia is a marker of vascular risk for microvascular and macrovascular complications in not only type 2 diabetes but also for those with impaired glucose tolerance (DiNicolantonio et al., 2015). Postprandial hyperglycemia causes vascular endothelial dysfunction by inducing oxidative stress responses that decrease NO bioavailability. Major sources of oxidative stress during postprandial hyperglycemia include the production of mitochondria-

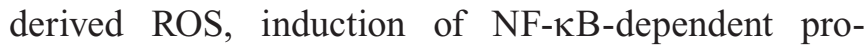
inflammatory enzymes and cytokines, and uncoupling of eNOS (Mah et al., 2012).

Alpha-glucosidase is an enzyme that regulates postprandial glucose levels by hydrolyzing carbohydrates into glucose in the small intestine (Tundis et al., 2010). 
Inhibition of the $\alpha$-glucosidase enzyme in the small intestine is an effective approach for the management of carbohydrate metabolic disorders, including type 2 diabetes mellitus (Olubomehin et al., 2013). Therefore, a natural product that has activity as an $\alpha$-glucosidase inhibitor becomes one of the main targets in searching for new compounds for the therapeutic management of diabetes (Alam et al., 2017).

Available studies on the antihyperglycemic effects of $C$. nutans were all performed in vitro. Lee et al. (2014) demonstrated that the methanol extracts of the leaves and stem of $C$. nutans exhibited $\alpha$-glucosidase inhibitory activity (13.57\% and $17.67 \%$, respectively) at the dose of $5 \mathrm{mg} / \mathrm{mL}$. Further, Wong et al. (2014) reported that treatment with a higher dose $(50 \mathrm{mg} / \mathrm{mL})$ of aqueous extract of $C$. nutans showed high levels of inhibition of $\alpha$-glucosidase activity $\left(88.2 \%, \mathrm{IC}_{50}: 30\right.$ $\mathrm{mg} / \mathrm{mL}$ ). In another recent report, Khoo et al. (2013) showed that extract from oven-dried leaves of $C$. nutans (70\% ethanol with sonication) inhibited $\alpha$-glucosidase activity up to $41 \%$ at a concentration of $5000 \mathrm{ppm}$, or approximately $5 \mathrm{mg} / \mathrm{mL}$.

In addition, a recent study by Alam et al. (2017) reported on the $\alpha$-glucosidase inhibitory activity of $C$. nutans methanol extract and its fraction at six different dilutions $(6.25,12.5,25,50,100$, and $200 \mu \mathrm{g} / \mathrm{mL})$. Butanol, ethyl acetate, and hexane fractions revealed similar $\alpha$-glucosidase inhibitory activities of $72.16 \%$ $\left(\mathrm{IC}_{50}: 37.47 \mu \mathrm{g} / \mathrm{mL}\right), 70.76 \%\left(\mathrm{IC}_{50}: 53.69 \mu \mathrm{g} / \mathrm{mL}\right)$, and $69.94 \%\left(\mathrm{IC}_{50}: 44.57 \mu \mathrm{g} / \mathrm{mL}\right)$, respectively, whereas the methanol extract showed moderate inhibitory activity at $58.21 \%\left(\mathrm{IC}_{50}: 61.39 \mu \mathrm{g} / \mathrm{mL}\right)$. The butanol fraction had significantly higher $\alpha$-glucosidase inhibitory activity, with an $\mathrm{IC}_{50}$ of $37.47 \mu \mathrm{g} / \mathrm{mL}$, which is close to the standard quercetin (positive control) compound $\left(\mathrm{IC}_{50}\right.$ : $38.54 \mu \mathrm{g} / \mathrm{mL}$ ).

Previous studies showed that $\alpha$-glucosidase inhibitory activity in extracts mostly involved flavonoid compounds (Alam et al., 2017; Fornari et al., 2012). Khoo et al. (2013) showed that application of the oven-dried method with sonication during $70 \%$ ethanol extraction resulted in greater levels of the phenolics, terpenoids, and sulfur-containing glucosides that contributed to the $\alpha$-glucosidase inhibitory activity. Based on data reported by Alam et al. (2017), the phytoconstituents that were identified from the methanol extract and four fractions of $C$. nutans included neophytadiene, squalene, lupeol, tocopherols, vanillic acid, syringic acid, myo-inositol, glycolic acid, butanedioic acid, 4-coumaric acid, and stigmasterol. Extensive literature searches document that the identified compounds were responsible for various biological activities, including acting as antioxidant, antidiabetic, anti-inflammatory, chemotherapeutic, and antihyperglycemic agents (Alam et al., 2017; Ow, Ieva, 2003). The structural orientation of the polyphenolic compound, owing to the lactones/ quinones or 4-oxo-pyran moiety, is responsible for the digestive enzyme inhibitory activity (Alam et al., 2017). Previous studies showed that phenolic compounds from C. nutans helped to reduce intestinal digestive enzyme activity and were able to oxidize body fat, owing to their thermogenic properties (Alam et al., 2017; Alterio et al., 2007), whereas the terpenoids and terpenes class of compounds can act as antihyperglycemic agents (Alam et al., 2017; Tundis et al., 2010).

In summary, based on previous studies, it appears that the methanol extract of $C$. nutans leaves is the best extract type for exhibiting $\alpha$-glucosidase inhibition activity, although the crude extract showed moderate activity. In addition, the butanol fraction from the methanol extract of $C$. nutans appears to show a greater inhibitory effect on $\alpha$-glucosidase (Alam et al., 2017).

\section{Antioxidant properties and effects on insulin resistance}

Many studies have provided evidence for the significant role of oxidative stress in insulin-resistant states such as type 2 diabetes, obesity, and metabolic syndrome (Pitocco et al., 2013). Oxidative stress occurs due to an imbalance between endogenous antioxidant systems and the generation of ROS. ROS overproduction has been reported to be an important trigger of insulin resistance and a contributing factor in the development of type 2 diabetes (Houstis et al., 2006), and previous studies have suggested that ROS and endothelial dysfunction play significant roles in the pathogenesis of diabetic vasculopathy (Schaffer et al., 2012). It has been shown that, in diabetes, the endothelium fails to produce adequate amounts of $\mathrm{NO}$, and blood vessels fail to relax in response to endothelium-dependent vasorelaxants such as acetylcholine, shear stress, and bradykinin (Ceriello et al., 2001). High levels of plasma glucose lead to increased mitochondrial formation of superoxide, a ROS that produces peroxynitrite when reacting with NO. It has been reported that peroxynitrite production is increased in the platelets of diabetic individuals (Ceriello et al., 2008). The levels of nitrotyrosine (a marker of ROS) in endothelial cells, myocytes, and fibroblasts significantly relate to the 
degree of cell death (Tannous et al., 1999) in the hearts of both diabetic patients and streptozotocin-induced diabetic rats (Frustaci et al., 2000). Peroxynitrite causes cellular damage through various mechanisms, including degradation of different biomolecules in the vascular endothelium, myocardium, and vascular smooth muscle and the depletion of tetrahydrobiopterin, the cofactor of eNOS for NO biosynthesis (Pitocco et al., 2013).

Free radical species can be neutralized through dismutation or reduction by endogenous antioxidants like superoxide dismutase (SOD) and catalase, as well as through direct scavenging or electron transfer by exogenous antioxidants such as vitamins $\mathrm{C}$ and $\mathrm{E}$ (Kohen, Niska, 2002). Pannangpetch et al. (2007) first reported the antioxidant activity of $C$. nutans where the ethanol extract $(50 \%)$ of the dried leaves exhibited a dose-dependent ability to scavenge 2,2-diphenyl-1picrylhydrazyl (DPPH), with an $\mathrm{IC}_{50}$ value of $110.4 \pm$ $6.59 \mu \mathrm{g} / \mathrm{mL}$. Although a $50 \%$ scavenging effect was achieved at a dose of $110 \mu \mathrm{g} / \mathrm{mL}$, the highest dose $(1000$ $\mu \mathrm{g} / \mathrm{mL}$ ) did not show complete DPPH inhibition but, rather, did show a moderate radical scavenging activity of $67.65 \%$. While the positive control, ascorbic acid $\left(\mathrm{IC}_{50}: 9.56 \pm 0.56 \mu \mathrm{g} / \mathrm{mL}\right.$ ), exhibited an almost complete radical scavenging effect, the DPPH scavenging efficacy of $C$. nutans was only 0.08 times that of ascorbic acid.

Yong et al. (2013) showed that the semipolar chloroform extract of $C$. nutans leaves demonstrated the most effective DPPH radical scavenging effect when compared to methanol and aqueous extracts, with high levels of antioxidant capacity of $7852.63 \pm 449.90 \mu \mathrm{g}$ $\mathrm{Teq} / \mathrm{g}$ extract at different concentrations $(12.5,25,50$, and $100 \mu \mathrm{g} / \mathrm{mL}$ ). The aqueous extract showed the lowest activity, with an antioxidant activity value of $864.11 \pm$ $73.49 \mu \mathrm{g} \mathrm{Teq} / \mathrm{g}$ extract. The antioxidant effect of the $C$. nutans extracts decreased in the order of chloroform $>$ methanol $>$ aqueous. In addition, the chloroform extract showed a greater galvinoxyl radical scavenging effect, with a high value of $12,248.82 \pm 173.50 \mu \mathrm{g}$ Teq $/ \mathrm{g}$ extract. However, that study also demonstrated that NO and hydrogen peroxide radical scavenging activities were lower than those of the positive controls ascorbic acid and quercetin (both at $100 \mu \mathrm{g} / \mathrm{mL}$ ), which exhibited more than $50 \%$ activities. The $\mathrm{IC}_{50}$ value and the antioxidant activity obtained in the study (aqueous extract) were similar to the first study on the antioxidant activity of $C$. nutans extract against DPPH (Pannangpetch et al., 2007).

In a different study, Arullappan et al. (2014) demonstrated that methanol, ethyl acetate, and petroleum ether extracts of $C$. nutans leaves exhibited greater DPPH radical scavenging activity, between $70 \%$ and $82 \%$, at higher concentration ranges of $4-10 \mathrm{mg} / \mathrm{mL}$. Similarly, Wong et al. (2014) showed that the aqueous extract of C. nutans at $10 \mathrm{mg} / \mathrm{mL}$ resulted in $60 \% \mathrm{DPPH}$ radical scavenging activity. Furthermore, the aqueous extract of the whole plant demonstrated metal chelating activity of up to $90 \%$ in a dose-dependent manner $(1-10 \mathrm{mg} / \mathrm{mL})$. Sulaiman et al. (2015) reported that chloroform and ethyl acetate extracts of $C$. nutans showed high levels of DPPH radical scavenging activity when compared to ethanol and hexane extracts. The hexane extract of $C$. nutans exhibited the greatest antioxidant activity (720 $\mathrm{mg}$ Teq/100 $\mathrm{g}$ extract) in the $\beta$-carotene bleaching assay, while the ethanol extract showed the lowest activity in this study. In contrast, when tested using an oxygen radical absorbance capacity (ORAC) assay, the ethanol extract demonstrated the most effective antioxidant activity ( $229.5 \mathrm{mMol} \mathrm{TE} / 100 \mathrm{~g}$ extract), followed by the ethyl acetate, chloroform, and hexane extracts. However, the study lacked positive controls in all their assays, and the authors did not properly indicate the concentration range used; therefore, it is difficult to determine the dose-dependent relationship.

Wanikiat et al. (2008) reported that the methanol extract of $C$. nutans showed a weak scavenging activity, with an $\mathrm{EC}_{50}$ of $240 \pm 15.3 \mathrm{mg} / \mathrm{mL}$, as compared to the positive control Trolox $\left(\mathrm{EC}_{50}: 7.5 \pm 2.3 \mu \mathrm{g} / \mathrm{mL}\right)$. Although the majority of DPPH radical scavenging of methanol extract showed a weak antioxidant activity, in vitro administration of $C$. nutans methanol extract (0.1$100 \mu \mathrm{g} / \mathrm{mL}$ ) dose-dependently suppressed $N$-formylmethionyl-leucyl-phenylalanine (fMLP)-induced superoxide anions in human neutrophils, with an $\mathrm{IC}_{50}$ of $24.3 \pm 3.1 \mu \mathrm{g} / \mathrm{mL}$. Although the positive control indomethacin $(0.01-100 \mu \mathrm{g} / \mathrm{mL})$ inhibits superoxide anions more effectively $\left(\mathrm{IC}_{50}: 0.82 \pm 0.2 \mu \mathrm{g} / \mathrm{mL}\right.$ ), the C. nutans inhibitory activity against fMLP-induced superoxide anions was significant and effective compared to untreated cells (Wanikiat et al., 2008).

Excessive production of reactive nitrogen species (RNS), which includes NO and peroxynitrite (ONOO-), has induced aberrant inflammation by causing oxidative and nitrosative or nitrative stress, lipid peroxidation, and disruption of cell membrane integrity. These processes can lead to the disruption of the mitochondrial electron transport chain (ETC) complexes that alter mitochondrial function, inducing mitochondrial leakage, with enhanced ROS production (Osto et al., 2008; Ott et al., 
2007; Preiser, 2012). Additionally, the aqueous extract of C. nutans showed the ability to scavenge NO in a dosedependent manner, with a moderate scavenging activity of $32.33 \pm 0.97 \%$ at $100 \mu \mathrm{g} / \mathrm{mL}$ (Yong et al., 2013). This moderate NO radical scavenging activity of $C$. nutans was supported by a recent finding that demonstrated that the whole aqueous extract of $C$. nutans scavenged NO in a concentration-dependent manner at a higher dose range of 1,5 , and $10 \mathrm{mg} / \mathrm{mL}$. The aqueous extract of $C$. nutans at a dose of $10 \mathrm{mg} / \mathrm{mL}$ showed more than $90 \%$ NO scavenging activity (Wong et al., 2014).

Furthermore, a recent report demonstrated that the antioxidant activity of $C$. nutans is associated with its ability to modulate the expression of various antioxidant genes (Sarega et al., 2016). Administration of aqueous and aqueous methanol leaf extracts of $C$. nutans upregulated the expression of SOD1, SOD2, catalase, glutathione reductase, and glutathione peroxidase. These then significantly increased hydroxyl radical scavenging activities and suppressed lipid peroxidation in rats fed a high-fat and high-cholesterol diet (Sarega et al., 2016). Additionally, both hexane and methanol leaf extracts showed the greatest DPPH, 2,2-azinobis(3-ethylbenzothiazoline-6-sulfonic acid) (ABTS), and ferric reducing ability (FRAP) radical scavenging activities because of the high amounts of phenolic compounds present in the extracts (Sarega et al., 2016). Generally, all studies indicated the antioxidant activities of $C$. nutans to be moderate, with efficacy of approximately $60-80 \%$ radical scavenging activity at higher concentration ranges (in $\mathrm{mg} / \mathrm{mL}$ ).

All except one study (Sarega et al., 2016) used the DPPH method to assess the antioxidant properties of $C$. nutans. The DPPH method has been reported to be an easy, fast, and reliable method that does not require a special device and reactions, as compared to other antioxidant assays (Aksoy et al., 2013). Based on the above data, it appears that aqueous and methanol leaf extracts of $C$. nutans showed significant antioxidant activity in different assays, including DPPH, NO scavenging, SOD, ABTS, and FRAP, when compared to other crude extracts, and both are suggested to be the best extracts of $C$. nutans for exhibiting antioxidant activity.

\section{Anti-inflammatory effects}

Inflammation is the body's protective response to infection or injury, but excessive inflammation can have detrimental effects and contributes to the progression of chronic and/or prolonged diseases such as atherosclerosis, rheumatoid arthritis, and systemic lupus erythematosus (Keane, Strieter, 2000).

Inflammation may promote vasculopathy by causing endothelial dysfunction. Endothelial dysfunction is defined as the failure of the vascular endothelium to subserve its normal role in vasodilatation and/or vascular homeostasis, and it commonly results from an imbalance between endothelium-derived contracting and relaxing factors (Rahman et al., 2007). Inflammation can modify the synthesis and degradation of vasodilators and vasoconstrictors, including $\mathrm{NO}$, and it impairs NO bioactivity. A previous study showed that rats treated with the NOS inhibitor N-nitro-L-arginine methyl ester (L-NAME) had higher blood pressure than control rats (Baylis et al., 1992). The study also demonstrated that inflammation downregulated NOS activity. In addition, c-reactive protein (CRP) and tumor necrosis factor (TNF) have been shown to reduce NO production by impairing the expression of eNOS mRNA (Verma et al., 2002; Yan et al., 2008). Further, a previous study showed that inhibition of TNF restored endothelial-dependent vasodilation in humans (MakiPetaja et al., 2006). Another cytokine, IL-17, has been demonstrated to cause endothelial dysfunction by activating Rho-kinase, which leads to phosphorylation of the inhibitory eNOS residue, threonine 495 (Nguyen et al., 2013). Inhibition of eNOS increases vascular tone (Baylis et al., 1992) and, consistent with this condition, Rho-kinase has been demonstrated to contribute to increased cerebral vascular tone in vivo.

Carrageenan-induced paw edema is an acute model of inflammation that is widely used for investigation of the anti-inflammatory effects of different compounds. Paw edema formation is a result of synergism between inflammatory mediators that increase blood flow and microvascular permeability (Wanikiat et al., 2008). This acute model involves two phases: The early phase observed at 1 hour is associated with the release of serotonin, histamine, and bradykinin and, to a minor extent, prostaglandins. The delayed phase (after 1 hour) is associated with polymorphonuclear (PMN) leucocyte infiltration and the continuation of prostaglandin generation (Di Rosa et al., 1971; Gilligan et al., 1994). The release of PMN leucocyte derived free radicals, NO, ROS, and pro-inflammatory cytokines such as IL-1 and TNF- $\alpha$ are associated with the delayed phase of carrageenan-induced inflammation (Halici et al., 2007; Nacife et al., 2004). 
The anti-inflammatory activity of the butanol fraction of C. nutans leaves has been reported to reduce carrageenaninduced edema (Kamarudin et al., 2017; Kittisiripornkul, Witthayasat, 1984). Studies have also shown that topical application of the whole-plant methanol extract of $C$. nutans $(3,6,9 \mathrm{mg} / 20 \mu \mathrm{L}$ acetone/ear) suppressed ethyl phenylpropiolate (EPP)-induced ear edema in rats in a significant concentration-dependent manner at all time points (15 $\mathrm{min}, 30 \mathrm{~min}, 60 \mathrm{~min}$, and $120 \mathrm{~min}$ ), and the highest dose of $C$. nutans $(9 \mathrm{mg} / 20 \mu \mathrm{L}$ acetone/ear) exhibited more edema inhibition than the positive control (indomethacin, $2 \mathrm{mg} / 20 \mu \mathrm{L}$ acetone/ear) at all time points (Kittisiripornkul, Witthayasat, 1984). Wanikiat et al. (2008) also reported that oral administration of the wholeplant methanol extract $(50,100$, and $200 \mathrm{mg} / \mathrm{kg}$ ) decreased carrageenan-induced edema in rat hind paws in a dosedependent manner, resulting in $30 \%, 49 \%$, and $59 \%$ inhibition, compared to the positive control (indomethacin, $20 \mathrm{mg} / \mathrm{kg}$ ), with 76\% inhibition. Pongphasuk et al. (2003) reported that treatment with the ethanol extract of $C$. nutans leaves (95\%) at the dose of $5 \mathrm{~g} / \mathrm{kg}$ showed $17 \%$ and $36 \%$ inhibition against carrageenan-induced paw edema at 3 and 6 hours, respectively.

Wanikiat et al. (2008) also reported that pretreatment with the methanol extract of $C$. nutans $\left(\mathrm{IC}_{50}: 2.7 \pm\right.$ $0.6 \mu \mathrm{g} / \mathrm{mL}$ ) suppressed fMLP-induced chemotaxis and chemokinesis in human neutrophils in a dose-dependent manner. The inhibition was significant when compared to the non-treated group, although there was less activity than in the positive control indomethacin $\left(\mathrm{IC}_{50}: 56.3 \pm\right.$ $3.5 \mathrm{ng} / \mathrm{mL}$ ).

Toll-like receptor (TLR)-4 is the first line of host defense against acute and chronic inflammation and is one of the key pro-inflammatory signaling receptors (Mai et al., 2016). Activation of TLR-4 by lipopolysaccharides (LPS) enhances the production of $\mathrm{NO}$ and inflammatory cytokines through activation of NF- $\kappa \mathrm{B}$ and IRF3, and the inhibition of TLR-4 activation may produce antiinflammatory effects because TLR-4 is the upstream receptor that activates both NF- $\mathrm{B}$ and IRF3 signaling, the hallmark of inflammation (Mai et al., 2016). It has been reported that pretreatment with $C$. nutans polar leaves extract (LP), non-polar leaves extract (LN), polar stem extract (SP), and non-polar stem extract (SN) at $1.5625-100 \mu \mathrm{g} / \mathrm{mL}$ significantly reduced the production of NO in RAW 264.7 macrophage and TLR-4 activation in TLR-4-transfected human embryonic kidney cells. In addition, the $C$. nutans polar leaves extract (1.5625-100 $\mu \mathrm{g} / \mathrm{mL}$ ) demonstrated anti-inflammatory activity against
LPS by suppressing TNF- $\alpha$, IFN- $\gamma$, IL-1 $\beta$, IL-6, IL12p40, and IL-17 production and inhibiting the activation of $\mathrm{p} 38$ mitogen-activated protein kinases (MAPK), extracellular signal regulated kinase (ERK), c-Jun-N-terminal kinase (JNK), NF- $\kappa \mathrm{B}$ p 65, and interferon regulatory factor 3 (IRF3) (Mai et al., 2016). LPS produces a powerful inflammatory response through activation of TLR-4, resulting in activation of NF- $\mathrm{KB}$ and the production of NO and inflammatory cytokines, including TNF- $\alpha$, IFN- $\gamma$, IL-1 $\beta$, IL-6, IL12p40, and IL-1.

Generally, studies on anti-inflammatory activities of $C$. nutans extracts in carrageenan-induced paw edema and EPP-induced ear edema have shown significant inhibitory effects on edema formation. It has been suggested that the $C$. nutans extract strongly inhibited the release and/or effects of histamine and serotonin that are released by inflammatory mediators during inflammation in an EPP model. C. nutans extract also affected the synthesis and release of mediators during both phases of the responses in carrageenan-induced rat paw edema. In addition, $C$. nutans extract was found to attenuate neutrophil chemokinesis in fMLPinduced chemotaxis and chemokinesis. Additionally, activation of neutrophils resulted in both intracellular and extracellular production of the radical superoxide (O2-), which may be attributed to distinct pools of nicotinamide adenine dinucleotide phosphate (NADPH) oxidase (Wanikiat et al., 2008). It was shown that fMLP triggered neutrophil O2- generation in a concentrationdependent manner, which was significantly inhibited by C. nutans extract. The suppression of the production of NO and cytokines (TNF- $\alpha$, IFN- $\gamma$, IL-1 $\beta$, IL-6, IL12p40 and IL-1) suggests that the anti-inflammatory activity of the $C$. nutans extract is associated with the inhibition of TLR-4 activation (Mai et al., 2016). C. nutans is suggested to exert its anti-inflammatory activity by preventing the activation of TLR-4 receptors, thus reducing the production of inflammatory cytokines and TLR-4 related inflammatory proteins. The methanolic extract of $C$. nutans leaves is suggested to show the best anti-inflammatory activity because of its significant effect in different assays in reducing carrageenaninduced paw edema and EPP-induced ear edema and in inhibiting fMLP-induced chemotaxis and chemokinesis.

\section{Antihyperlipidemic effects}

Dyslipidemia is one of the major risk factors for cardiovascular disease in diabetes mellitus. The 
characteristic features of diabetic dyslipidemia are low levels of high-density lipoprotein cholesterol (HDL-C), high plasma triglycerides (TG), and increased levels of small dense low-density lipoprotein cholesterol (LDL-C) (Mooradian, 2009). The HDL-C particles induce the removal of cholesterol from cells, including those in atherosclerotic plaques, and carry them to the liver. However, the mechanism by which HDL-C confers protection from atherosclerosis involves more than just reverse cholesterol transport (Bitzur et al., 2009). HDL-C particles seem to have anti-inflammatory and antioxidant properties, inhibiting the oxidation of LDL-C and the expression of cellular adhesion molecules as well as monocyte recruitment (Bitzur et al., 2009).

High TG levels are the markers for several types of atherogenic lipoproteins. Hypertriglyceridemic states are associated with increased very low-density lipoprotein cholesterol (VLDL-C) production and delayed VLDL-C clearance from circulation (Talayero, Sacks, 2011). Insulin resistance is believed to contribute to this atherogenic dyslipidemia by increasing the hepatic secretion of VLDL-C and other apolipoprotein (apo) B-containing lipoprotein particles as a result of increased free fatty acid flux to the liver (Bitzur et al., 2009). This may also be the result of a diminished suppressive effect of insulin on apoB secretion, either at the level of the regulation of apoB degradation or through inhibition of microsomal TG transfer protein activity (Bitzur et al., 2009; Malmstrom et al., 1997).

Through the action of the cholesterol ester transfer protein, TG are transferred from VLDL-C to HDL-C, creating TG-rich HDL-C particles, which are hydrolyzed by hepatic lipase and rapidly cleared from the plasma (Bitzur et al., 2009). A similar cholesterol ester protein-mediated transfer of TG from VLDL-C to LDL-C contributes to the formation of small dense LDL-C particles. Similar to oxidized LDL (Ox-LDL), these cholesterol-enriched, TG-poor species are subject to endothelial accumulation and uptake by macrophages to form foam cells (Talayero, Sacks, 2011).

Hypercholesterolemia is a lipoprotein metabolic disorder characterized by altered metabolism of cholesterol, which promotes the production of ROS through modulation of the activities of enzymes such as NADPH oxidase and xanthine oxidase. The altered activities of these enzymes have been demonstrated to increase endothelial superoxide anion production, resulting in JNK-mediated inactivation of endotheliumderived $\mathrm{NO}$ and subsequent increases in oxygen radical production and inflammation (Osto et al., 2008; Sarega et al., 2016).

Hypercholesterolemia leads to accumulation and oxidation of LDL-C within the intima of the vessel wall and causes endothelial dysfunction (Osto et al., 2008; Sarega et al., 2016). Cholesterol, in particular LDL-C, is generally considered a major contributor to atherosclerosis susceptibility. LDL-C and other lipoproteins cross the endothelial cell via vascular transport, may be modified by oxidation, aggregation, or glycation, and are associated with proteoglycans or are incorporated into immuno-complexes (Rahman et al., 2007). LDL-C molecules are oxidatively modified at the subendothelial space into ROS that are generated by macrophages, endothelial cells, and smooth muscle cells (Rahman et al., 2007). Ox-LDL facilitates monocyte recruitment, activation, and differentiation to become larger macrophages, which bind and internalize Ox-LDL particles with cholesteryl ester accumulation and then become foam cells. Foam cells stimulate inflammatory mediators and alter both the structure and function of endothelial cells (Rahman et al., 2007). Ox-LDL increases the adhesion of circulating monocytes to damaged endothelium, increasing their migration into the vascular intima (Baumgartner-Parzer et al., 1995; Tesfamariam et al., 1991). Ox-LDL also decreases NO production by reducing NO synthase (Wautier, Guillausseau, 2001), thus contributing to defective vasodilatation.

Sarega et al. (2016) reported that in rats fed a highfat and high-cholesterol diet, the methanol extract of C. nutans leaves and phenolic-rich aqueous at doses of 125,250 , and $500 \mathrm{mg} / \mathrm{kg} /$ day $/$ rat exhibited good antihyperlipidemic activity that was comparable to the lipid-lowering drug simvastatin (10 $\mathrm{mg} / \mathrm{kg} /$ day $)$. After 7 weeks of treatment, the aqueous and methanol extract treated groups showed improvement in their lipid profiles, including reduced total cholesterol (TC), TG, LDL-C, and VLDL-C levels and increased HDL-C levels. The data showed that the methanol and aqueous extracts dose-dependently reduced TC levels $(28 \%$ and $18 \%$ reduction, respectively) at the dose of $500 \mathrm{mg} / \mathrm{kg}$. The methanol extract dose-dependently decreased both VLDL-C and TG levels and increased levels of HDL-C but not LDL-C, whereas the aqueous extract showed dose-dependent effects on TG and LDL-C but had no effect on HDL-C and VLDL-C levels. Further, at the highest dose of $500 \mathrm{mg} / \mathrm{kg}$, improvements were seen in TG, HDL-C, and VLDL-C levels with comparable efficacy to simvastatin treatment. 
Recently, Abdulwahid et al. (2017) reported the effects of methanol extract of $C$. nutans at different doses $(500,1000$, and $1500 \mathrm{mg} / \mathrm{kg})$ on lipid profiles in diet-induced obese mice. Their results showed that plasma TC levels were reduced in C. nutans treatment groups when compared to high-fat diet control mice.

It has been suggested that plant polyphenols may reduce plasma cholesterol by decreasing cholesterol absorption, forming insoluble precipitates of cholesterol, and decreasing bile acid-induced micellar solubility (Abdulwahid et al., 2017; Anandh Babu et al., 2006). Sarega et al. (2016) also reported that weight loss and antihyperlipidemic activities of $C$. nutans were associated with multiple phenolic compounds present in the extract, with protocatechuic acid being the most abundant. It can be hypothesized that the synergy between phenolic compounds and their ability to upregulate various hepatic antioxidant genes that suppress oxidative stress mainly contribute to the antihyperlipidemic properties of $C$. nutans. Thus, the hypocholesterolemic activity of the polyphenols in C. nutans could be due to decreased cholesterol absorption, enhanced cholesterol excretion, and inhibition of cholesterol biosynthesis (Abdulwahid et al., 2017). Based on previous studies, it appears that the methanol extract of $C$. nutans has better antihyperlipidemic effects than the aqueous extract.

\section{CONCLUSION}

Scientific findings, predominantly experimental studies, have shown that $C$. nutans extracts possess antihyperglycemic, antioxidant, anti-inflammatory, and antihyperlipidemic effects. C. nutans extracts possess significant antihyperglycemic properties, as demonstrated by its $\alpha$-glucosidase inhibitory activity. In terms of antioxidant properties, it has shown greater DPPH radical scavenging activity in a dose-dependent manner. It also upregulated the expression of SOD1, SOD2, glutathione reductase, and glutathione peroxidase and showed the greatest effects on ABTS and FRAP radical scavenging. C. nutans have significant anti-inflammatory properties, as shown by its effect in both the rat carrageenan-induced paw edema model and the EPP-induced rat ear edema model. For antihyperlipidemic studies, C. nutans extracts improved the lipid profile in rats. Phenolic compounds also reduced hyperlipidemia-induced oxidative stress. With these pharmacological properties, C. nutans has the potential to reduce the severity of or prevent diabetic cardiovascular complications, as these properties can act synergistically to reduce diabetic vasculopathy. Therefore, future studies on C. nutans in diabetes should assess its potential effects as an anti-atherosclerosis agent to reduce cardiovascular complications in diabetes. The present review provides the information, groundwork, and important directions for researchers in conducting further in vivo, in vitro, and clinical investigations of $C$. nutans in diabetic cardiovascular related studies.

\section{ACKNOWLEDGMENT}

We thank Universiti Sains Malaysia for Short term grant (304.PPSP.6315096) and Bridging grant (304. PPSP.6316127) for funding studies related to C. nutans.

\section{DECLARATION OF INTEREST STATEMENT}

The authors report no conflicts of interest.

\section{REFERENCES}

Abdulwahid SJ, Ebrahimi M, Goh Y, Adeyemi KD, Ismail H. Methanolic extract of Clinacanthus nutans leaves can alter adipocytes cellularity, inflammation, and acetyl cholinesterase activity in male obese mice. J Obes Weight Loss Ther. 2017;7:336.

Aksoy L, Kolay E, Agilonu Y, Aslan Z, Kargioglu M. Free radical scavenging activity, total phenolic content, total antioxidant status, and total oxidant status of endemic Thermopsis turcica. Saudi J Biol Sci. 2013;20(3):235-239.

Alam MA, Zaidul ISM, Ghafoor K, Sahena F, Hakim MA, Rafii MY, Abir HM, Bostanudin MF, Perumal V, Khatib A et al. In vitro antioxidant and, $\alpha$-glucosidase inhibitory activities and comprehensive metabolite profiling of methanol extract and its fraction from Clinacanthus nutans. BMC Complement Altern Med. 2017;17(1):181.

Alterio AA, Fava DAF, Navarro F. Interaction of the daily ingestion of green tea (Camella sinensis) in the cellular metabolism and the adipose cell promoting emagrecimento. Rev Bras Obes Nut Emag. 2007;1(3):27-37.

Anandh Babu PV, Sabitha KE, Shyamaladevi CS. Green tea extract impedes dyslipidemia and development of cardiac dysfunction in streptozotocin-diabetic rats. Clin Exp Pharmacol Physiol. 2006;33(12):1184-1189.

Arullappan S, Rajamanickam P, Thevar N, Kodimani CC. In vitro screening of cytotoxic, antimicrobial and antioxidant 
activities of Clinacanthus nutans (Acanthaceae) leaf extracts. Trop J Pharm Res. 2014;13(9):1455-1461.

Baumgartner-Parzer SM, Wagner L, Pettermann M, Gessel A, Waldha üsi W. Modulation by high glucose of adhesion molecule expression in cultured endothelial cells. Diabetologia. 1995;38(11):1367-1370.

Baylis C, Mitruka B, Deng A. Chronic blockade of nitric oxide synthesis in the rat produces systemic hypertension and glomerular damage. J Clin Invest. 1992;90(1):278-281.

Bitzur R, Cohen H, Kamari Y, Shaish A, Harats D. Triglycerides and HDL Cholesterol: Stars or second leads in diabetes? Diabetes Care. 2009;32(2):373-377.

Boden $\mathrm{G}$. Free fatty acids, insulin resistance and type 2 diabetes mellitus. Proc Assoc Am Physicians. 1999;111(3):241-248.

Ceriello A, Esposito K, Piconi L, Ihnat MA, Thorpe JE, Testa R, Boemi M, Giugliano D et al. Oscillating glucose is more deleterious to endothelial function and oxidative stress than mean glucose in normal and type 2 diabetic patients. Diabetes. 2008;57(5):1349-1354.

Ceriello A, Mercuri F, Quagliaro L, Assaloni R, Motz E, Tonutti, L, Taboga $\mathrm{C}$ et al. Detection of nitrotyrosine in the diabetic plasma: Evidence of oxidative stress. Diabetologia. 2001;44(7):834-838.

Creager MA, Lüscher TF, Cosentino F, Beckman JA. Diabetes and vascular disease pathophysiology, clinical consequences, and medical therapy: part I. Circulation. 2003;108(13): 1527-1532.

Daduang S, Uawonggul N, Watson R, Preedy V. Herbal therapies of snake and insect bites in Thailand. Bot. Med. Clin. Pract. 2008;814-822.

Di Rosa M, Giroud, JP, Willoughby DA. Studies on the mediators of the acute inflammatory response induced in rats in different sites by carrageenan and turpentine. J Pathol. 1971;104(1):15-29.

DiNicolantonio JJ, Bhutani J, O'Keefe JH. Acarbose: safe and effective for lowering postprandial hyperglycaemia and improving cardiovascular outcomes. Open Heart. 2015;2(1):e000327.

Dresner A, LaurentD, Marcucci M. Effects of free fatty acids on glucose transport and IRS-1-associated phosphatidyllinositol 3-kinase activity. J Clin Invest. 1999;103(2):253-259.

Fornari T, Vicente G, Vàzquez E, Garcìa-Risco MR, Reglero G. Isolation of essential oil from different plants and herbs by supercritical fluid extraction. J Chromatogr A. 2012;1250: 34-48.

Frustaci A, Kajstura J, Chimenti C, Jakoniuk I, Leri A, Maseri A, Nadal-Ginard B, Anversa P et al. Myocardial cell death in human diabetes. Circ. Res. 2000;87(12):1123-1132.

Funk SD, Yurdagul AJr, Orr AW. Hyperglycemia and endothelial dysfunction in atherosclerosis: lessons from type1 diabetes. Int J Vasc Med. 2012; 2012:ID569654.

Gilligan JP, Lovato SJ, Erion MD, Jeng AY. Modulation of carrageenan- induced hind paw edema by substance P. Inflammation. 1994;18(3):285-292.

Griffin ME, Marcucci MJ, Cline GW. Free fatty acid-induced insulin resistance is associated with activation of protein kinase $\mathrm{C}$ theta and alteration in the insulin signalling cascade. Diabetes. 1999;48(6):1270-1274.

Halici Z, Dengiz GO, Odabasoglu F, Suleyman H, Cadirci E, Halici $M$ et al. Amiodarone has anti-inflammatory and anti-oxidative properties: an experimental study in rats with carrageenan-induced paw edema. Eur J Pharmacol. 2007;566(1/3):215-221.

Ho SY, Tiew WP, Priya M, Mohamed SAS, Gabriel AA. Phytochemical analysis and antibacterial activity of methanolic extract of Clinacanthus nutans leaf. Int J Drug Dev Res. 2013;5(3):349-355.

Houstis N, Rosen ED, Lander ES. Reactive oxygen species have a causal role in multiple forms of insulin resistance. Nature. 2006;440(7086):944-948.

Huang D, Guo W, Gao J, Chen J, Olatunji J. Clinacanthus nutans (Burm. f.) Lindau ethanol extract inhibits hepatoma in mice through upregulation of the immune response. Molecules. 2015;20(9):17405-17428.

Inoguchi T, Li P, Umeda F. High glucose level and free fatty acid stimulate reactive oxygen species production through protein kinase $\mathrm{C}$-dependent activation of NAD(P)H oxidase in cultured vascular cells. Diabetes. 2000;49(11):1939-1945.

Kamarudin MNA, Sarker MMR, Kadir HA, Ming LC. Ethnopharmacological uses, phytochemistry, biological activities, and therapeutic application of Clinacanthus nutans (Burm. f.) Lindau: A comprehensive review. J Etnopharmacol. 2017;206;245-266.

Keane MP, Strieter RM. Chemokine signaling in inflammation. Crit Care Med. 2000;28(4):13-26.

Kelley DE, Simoneau JA. Impaired free fatty acid utilization by skeletal muscle in non-insulin-dependent diabetes mellitus. J Clin Invest. 1994; 94(6):2349-2356. 
Khoo LW, Mediani A, Zolkeflee NKZ, Leong SW, Ismail IS, Khatib A, Shaari K, Abas F et al. Phytochemical diversity of Clinacanthus nutans extracts and their bioactivity correlations elucidated by NMR based metabolomics. Phytochem Lett. 2015;14:123-133.

King GL. The role of hyperglycemia and hyperinsulinemia in causing vascular dysfunction in diabetes. Ann Med. 1996;28(5):427-432.

Kittisiripornkul S, Witthayasat, K. The anti-inflammatory action and toxicological studies of extracts from Clinacanthus nutans (Master thesis). Mahidol University, Bangkok, Thailand.1984.

Kohen R, Nyska A. Oxidation of biological systems: oxidative stress phenomena, antioxidants, redox reactions, and methods for their quantification. Toxicol Pathol. 2002;30(6):620-650.

Kolka CM, Bergman RN. The endothelium in diabetes: its role in insulin access and diabetic complications. Rev Endocr Metab Disord. 2013;14(1):13-19.

Kubes P, Suzuki M, Granger DN. Nitric oxide: an endogenous modulator of leukocyte adhesion. Proc Natl Acad Sci. USA. 1991;88(11):4651-4655.

Kunsorn P, Ruangrungsi N, Lipipun V, Khanboon A, Rungsihirunrat K. The identities and anti-herpes simplex virus activity of Clinacanthus nutans and Clinacanthus siamensis. Asian Pac J Trop Biomed. 2013;3(4):284-290.

Lee SY, Mediani A, AH NA, Abu Bakar Sajak A, Abas F. Antioxidant and $\alpha$-glucosidase inhibitory activities of the leaf and stem of selected traditional medicinal plants. Int Food Res J. 2014;21(1)165-172.

Mah E, Bruno RS. Postprandial hyperglycemia on vascular endothelial function: mechanism and consequences. Nutr Res. 2012;32(10):727-740.

Mai CW, Yap IKS, Kho MT, Ismail NH, Yusoff KM, Shaari K, Chin SY, Lim SHE et al. Mechanisms underlying the antiinflammatory effects of Clinacanthus nutans Lindau extracts: inhibition of cytokine production and Toll-like receptor-4 activation. Front Pharmacol. 2016;7:7.

Maki-Petaja KM, Hall FC, Booth AD. Rheumatoid arthritis is associated with increased aortic pulse-wave velocity, which is reduced by anti-tumor necrosis factor- $\alpha$ therapy. Circulation. 2006;114(11):1185-1192.

Malmstrom R, Packard CJ, Caslake M, Bedford D, Stewart P, Yki-Jarvinen H, Shepherd J, Taskinen MR et al. Defective regulation of triglyceride metabolism by insulin in the liver in NIDDM. Diabetologia. 1997;40(4):454-462.
Mokhtar SS, Vanhoutte PM, Leung SWS, Suppian R, Yusof MI, Rasool AHG et al. Reduced nitric oxide- mediated relaxation and endothelial nitric oxide synthase expression in the tail arteries of streptozotocin-induced diabetic rats. Eur J Pharm. 2016; 773:78-84.

Mooradian AD. Dyslipidemia in type 2 diabetes mellitus. Nat Clin Pract Endocrinol Metab. 2009;5(3):150-159.

Nacife VP, Soeiro MN, Gomes RN, D'Avila H, CastroFaria Neto HC, Meirelles MN et al. Morphological and biochemical characterization of macrophages activated by carrageenan and lipopolysaccharide in vivo. Cell Struct Funct. 2004;29(2):27-34.

Nguyen H, Chiasson VL, Chatterjee P, Kopriva SE, Young KJ, Mitchell BM et al. Interleukin-17 causes Rho- kinasemediated endothelial dysfunction and hypertension. Cardiovasc Res. 2013;97(4):696-704.

Olubomehin OO, Abo KA, Ajaiyeoba EO. Alpha-amylase inhibitory activity of two Anthocleista species and in vivo rat model antidiabetic activities of Anthocleista djalonensis extracts and fractions. J Ethnopharmacol. 2013;146(3): 811-814.

Osto E, Matter CM, Kouroedov A. c-Jun N-Terminal kinase 2 deficiency protects againts hypercholesterolemia-induced endothelial dysfunction and oxidative stress. Circulation. 2008; 118(20):2073-2080.

Ott M, Gogvadze V, Orrenius S, Zhivotovsky B. Mitochondria, oxidative stress and cell death. Apoptosis. 2007;12(5): 913-922.

Ow YY, Ieva S. Gallic acid and gallic acid derivatives: effects on drug metabolizing enzymes. Curr Drug Metab. 2003;4(3):241-248.

Pannangpetch P, Laupattarakasem P, Kukongviriyapan V, Kukongviriyapan U, Kongyingyoes B, Aromdee C et al. Antioxidant activity and protective effect against oxidative hemolysis of Clinacanthus nutans (Burm. f) Lindau. Songklanakarin J. Sci. Technol. 2007;29(1):1-9.

Pitocco D, Tesauro M, Alessandro R, Ghirlanda G, Cardillo C. Oxidative stress in diabetes: Implications for vascular and other complications. Int J Mol Sci. 2013;14(11):21525-21550.

Pongphasuk N, Khunkitti W, Chitcharoenthum M. Antiinflammatory and Analgesic activities of the extract from Garcinia mangostana Linn. III WOCMAP Congr. Med. Aromat. Plants-Vol. 6: Tradit. Med. Nutraceuticals. 2003;680:125-130. 
Preiser J-C. Oxidative stress. J. Parenter Enter Nutr. 2012;36(2):147-154.

Radomski MW, Palmer RM, Moncada S. The role of nitric oxide and cGMP in platelet adhesion to vascular endothelium. Biochem Biophys Res Commun. 1987;148(3):1482-1489.

Rahman S, Rahman T, Ismail AA, Rashid AAA. Diabetesassociated macrovasculopathy: pathophysiology and pathogenesis. Diabetes Obes Metab. 2007;9(6):767-780.

Sakdarat S, Shuyprom A, Ayudhya TDN, Waterman PG, Karagianis G. Chemical composition investigation of the Clinacanthus nutans Lindau leaves. Thai J. Phytopharm. 2006;13(2):13-24.

Sangkitporn S, Chaiwat S, Balachandra K, Na-Ayudhaya TD, Bunjob M, Jayavasu C et al. Treatment of herpes zoster with Clinacanthus nutans (bi phaya yaw) extract. J. Med. Assoc. Thai. Chotmaihet thangphaet. 1995;78(11):624-627.

Sarega N, Imam MU, Ooi D-J, Chan KW, Md Esa N, Zawawi $\mathrm{N}$, Ismail $\mathrm{M}$ et al. Phenolic rich extract from Clinacanthus nutans attenuates hyperlipidemia- associated oxidative stress in rats. Oxid Med Cell Longev. 2016;2016:ID 4137908.

Sarkar R, Meinberg EG, Stanley JC. Nitric oxide reversibly inhibits the migration of cultured vascular smooth muscle cells. Circ Res. 1996;78:225-230.

Schaffer SW, Jong CJ, Mozaffari M. Role of oxidative stress in diabetes-mediated vascular dysfunction: Unifying hypothesis of diabetes revisited. Vascul. Pharmacol. 2012;57(5/6): 139-149.

Sharma A, Bernatchez PN, de Haan JB. Targeting endothelial dysfunction in vascular complications associated with diabetes. Int J Vasc Med. 2012;2012:750126.

Shim SY, Aziana I, Khoo BY. Perspective and insight on Clinacanthus nutans Lindau in traditional medicine. Int $\mathrm{J}$. Integr. Biol. 2013;14(1):7-9.

Steinberg HO, Tarshoby M, Monestel R. Elevated circulating free fatty acid levels impair endothelium-dependent vasodilation. J Clin Invest. 1997;100:1230-1239.

Sulaiman ISC, Basri M, Chan KW, Ashari SE, Masoumi $\mathrm{HRF}$, Ismail, $\mathrm{M}$ et al. In vitro antioxidant, cytotoxic and phytochemical studies of Clinacanthus nutans Lindau leaf extracts. Afr J Pharm Pharmacol. 2015;9(34):861-874.
Talayero BG, Sacks FM. The role of triglycerides in atherosclerosis. Curr Cardiol Rep. 2011;13(6): 544-552.

Tannous M, Rabini RA, Vignini A, Moretti N, Fumelli P, Zielinski B, Mazzanti, L, Mutus B et al. Evidence for iNOSdependent peroxynitrite production in diabetic platelets. Diabetologia. 1999;42(5):539-544.

Tesfamariam B, Brown ML, Cohen RA. Elevated glucose impaired endothelium-dependent relaxation by activating protein kinase C. J Clin Invest. 1991;87(5):1643-1648.

Tundis R, Loizzo M, Menichini F. Natural products as a-amylase and $\alpha$-glucosidase inhibitors and their hypoglycemic potential in the treatment of diabetes: an update. Mini Rev Med Chem. 2010;10(4):315-331.

Uawonggul N, Thammasirirak S, Chaveerach A, Chuachan C, Daduang J, Daduang, S et al. Plant extract activities against the fibroblast cell lysis by honey bee venom. J. Med. Plants Res. 2011;5(1):1978-1986.

Verma S, Wang C, Li S. A self-fullling prophecy: C-reactive protein attenuates nitric oxide production and inhibits angiogenesis. Circulation. 2002;106(8):913-919.

Wanikiat P, Panthong A, Sujayanon P, Yoosook C, Rossi AG, Reutrakul $\mathrm{V}$ et al. The anti-inflammatory effects and the inhibition of neutrophil responsiveness by Barleria lupulina and Clinacanthus nutans extracts. J Ethnopharmacol. 2008;116(2):234-244.

Wautier JL, Guillausseau PJ. Advanced glycation end products, their receptors and diabetic angiopathy. Diabetes Metab. 2001;27(5 Pt 1):535-542.

Wong F-C, Yong, A-L, Ting EP-S, Khoo S-C, Ong H-C, Chai T-T et al. Antioxidant, metal chelating, anti-glucosidase activities and phytochemical analysis of selected tropical medicinal plants. Iran J Pharm Res. 2014;13(4):1409-1415.

Yan G, You B, Chen S, Liao JK, Sun J. Tumor necrosis factor- $\alpha$ downregulates endothelial nitric oxide synthase mRNA stability via translation elongation factor $1-\alpha 1$. Circ Res. 2008;103(6):591-597.

Yong YK, Tan JJ, Teh SS, Mah SH, Ee GCL, Chiong HS, Ahmad $\mathrm{Z}$ et al. Clinacanthus nutans extracts are antioxidant with antiproliferative effect on cultured human cancer cell lines. J Evid Based Complement Altern Med. 2013;2013:ID462751

Received for publication on $10^{\text {th }}$ October 2018 Accepted for publication on $01^{\text {st }}$ February 2019 